\title{
Editorial to the special issue on "Molecular Genetics of Developmental Eye Disorders"
}

\author{
Nicola Ragge $^{1,2} \cdot$ Patrick Calvas $^{3} \cdot$ Nicolas Chassaing $^{3}$
}

Published online: 5 August 2019

(c) Springer-Verlag GmbH Germany, part of Springer Nature 2019

At a landmark time in the expansion of our knowledge of genetics and genomics, we have had the privilege of gathering together articles that provide a unique insight into our current knowledge of eye development and the efforts that have helped us reach this point. We have commissioned articles that describe the genetic basis of structural eye disorders, in other words approaching from the phenotype to the genotype; animal models that have advanced our understanding of mechanisms of eye development; detailed analyses of pathways, and a glimpse towards the future in therapeutic approaches. Related free papers have been incorporated into the issue to add context.

As well as consolidating knowledge in the area, we have aimed to capture the interest of emergent physicians and researchers into this fascinating area and inspire studies to further understand the mechanisms involved in eye development and to create novel therapeutic strategies. It is now clear that increasing our understanding of the genetic basis of developmental eye disorders is now only attainable through enhanced communication between clinicians and scientists. It is without doubt this collaborative approach that encompasses the spirit and content of this Special Issue.

We owe a huge debt of gratitude to all the contributors for their immense hard work and dedication in carefully crafting these excellent articles. We also thank the Editors and Production Staff at Springer for their support in creating this Special Issue, which we hope that we will serve the needs of clinicians and scientists and ultimately our patients.
This Special Issue is dedicated to the families affected by Developmental Eye Disorders, whose courage in living with these conditions is an inspiration to us all.

Nicola Ragge

nragge@brookes.ac.uk

1 Faculty of Health and Life Sciences, Oxford Brookes University, Oxford OX3 0BP, UK

2 West Midlands Regional Clinical Genetics Service and Birmingham Health Partners, Birmingham Women's and Children's NHS Foundation Trust, Birmingham B15 2TG, UK

3 INSERM1056, Université de Toulouse, Centre de Référence des Anomalies Rares en Génétique Ophtalmologique, Service de Génétique Médicale, Centre Hospitalier Universitaire de Toulouse, Toulouse, France 